EPJ manuscript No.

(will be inserted by the editor)

\title{
Recent breakthroughs in Skyrme-Hartree-Fock-Bogoliubov mass formulas
}

\author{
S. Goriely ${ }^{1}$, N. Chamel ${ }^{\mathrm{a} 1}$, and J. M. Pearson ${ }^{2}$ \\ 1 Institut d'Astronomie et d'Astrophysique, Université Libre de Bruxelles, CP226, 1050 Brussels, Belgium \\ 2 Déptartement de Physique, Université de Montréal, Montréal (Qc) H3C 3J7, Canada \\ Received: date / Revised version: date
}

\begin{abstract}
We review our recent achievements in the construction of microscopic mass tables based on the Hartree-Fock-Bogoliubov method with Skyrme effective interactions. In the latest of our series of HFBmass models, we have obtained our best fit ever to essentially all the available mass data, by treating the pairing more realistically than in any of our earlier models. The rms deviation on the 2149 measured masses of nuclei with $N$ and $Z \geq 8$ has been reduced for the first time in a mean field approach to 0.581 $\mathrm{MeV}$. With the additional constraint on the neutron-matter equation of state, this new force is thus very well-suited for the study of neutron-rich nuclei and for the description of astrophysical environments like supernova cores and neutron-star crusts.
\end{abstract}

PACS. 21.10.Dr Binding energies and masses - 21.30.Fe Forces in hadronic systems and effective interactions - 21.60.Jz Hartree-Fock and random-phase approximations - 26.60.Gj Neutron star crust

\section{Introduction}

Nuclear astrophysics applications require the knowledge of various nuclear properties (nuclear masses, nuclear level densities (NLD), optical potentials, $\gamma$-ray strength functions, etc.) that cannot be measured experimentally in the foreseeable future. In order to make reliable extrapolations of these quantities far from the domain covered by experimental data, we have developed a series of nuclear-mass models based on the Hartree-Fock-Bogoliubov (HFB) meth with Skyrme and contact-pairing forces, together with phenomenological Wigner terms and correction terms for the spurious collective energy. The model parameters are fitted to essentially all the available atomic mass data, requiring that the model reproduce several properties of uniform asymmetric nuclear matter determined by microscopic calculations with realistic nucleon-nucleon potentials. With these nuclear-matter constraints, our models can be reliably applied to study astrophysical environments such as supernova cores and neutron star crusts 1 . In particular, model HFB-9 [2] and all later models constrained the underlying Skyrme force to fit the equation of state of neutron matter, as calculated by Friedman and Pandharipande [3] for realistic two- and three-nucleon forces.

In this paper, we present our latest models, HFB-16 [4] and HFB-17 [5], in which we have imposed the additional constraint of reproducing as a function of density the ${ }^{1} S_{0}$ pairing gaps of uniform asymmetric nuclear matter. This

\footnotetext{
a Conference presenter
}

latter constraint is not only of prime importance for reliable investigations of a possible superfluid phase in the inner crust of neutron stars, but also turns out to significantly improve the accurary of the mass fit.

\section{The Hartree-Fock-Bogoliubov mass formulas}

The Hartree-Fock-Bogoliubov (HFB) mass models HFB16 and HFB-17 are based on the conventional Skyrme force of the form

$$
\begin{aligned}
v^{\mathrm{Sky}}\left(\boldsymbol{r}_{\boldsymbol{i}}, \boldsymbol{r}_{\boldsymbol{j}}\right)= & t_{0}\left(1+x_{0} P_{\sigma}\right) \delta\left(\boldsymbol{r}_{i j}\right) \\
& +\frac{1}{2} t_{1}\left(1+x_{1} P_{\sigma}\right) \frac{1}{\hbar^{2}}\left[p_{i j}^{2} \delta\left(\boldsymbol{r}_{i j}\right)+\delta\left(\boldsymbol{r}_{i j}\right) p_{i j}^{2}\right] \\
& +t_{2}\left(1+x_{2} P_{\sigma}\right) \frac{1}{\hbar^{2}} \boldsymbol{p}_{i j} \cdot \delta\left(\boldsymbol{r}_{i j}\right) \boldsymbol{p}_{i j} \\
& +\frac{1}{6} t_{3}\left(1+x_{3} P_{\sigma}\right) \rho(\boldsymbol{r})^{\gamma} \delta\left(\boldsymbol{r}_{i j}\right) \\
& +\frac{\mathrm{i}}{\hbar^{2}} W_{0}\left(\boldsymbol{\sigma}_{\boldsymbol{i}}+\boldsymbol{\sigma}_{\boldsymbol{j}}\right) \cdot \boldsymbol{p}_{i j} \times \delta\left(\boldsymbol{r}_{i j}\right) \boldsymbol{p}_{i j} \quad, \quad \text { (1) }
\end{aligned}
$$

where $\boldsymbol{r}_{i j}=\boldsymbol{r}_{i}-\boldsymbol{r}_{j}, \boldsymbol{r}=\left(\boldsymbol{r}_{i}+\boldsymbol{r}_{j}\right) / 2, \boldsymbol{p}_{i j}=-\mathrm{i} \hbar\left(\boldsymbol{\nabla}_{i}-\nabla_{j}\right) / 2$ is the relative momentum, and $P_{\sigma}$ is the two-body spinexchange operator. The contact pairing force acts only between nucleons of the same charge state $q(q=n$ or $p$ for neutron or proton, respectively) and is given by

$$
v_{q}^{\mathrm{pair}}\left(\boldsymbol{r}_{\boldsymbol{i}}, \boldsymbol{r}_{\boldsymbol{j}}\right)=v^{\pi q}\left[\rho_{n}(\boldsymbol{r}), \rho_{p}(\boldsymbol{r})\right] \delta\left(\boldsymbol{r}_{i j}\right)
$$


where $v^{\pi q}\left[\rho_{n}, \rho_{p}\right]$ is a functional of the nucleon densities.

To the HFB energy calculated for the Skyrme force (1) and the pairing force (2) are added two phenomenological corrections: (i) the Wigner energy 6, 7

$$
\begin{aligned}
& E_{W}=V_{W} \exp \left\{-\lambda\left(\frac{N-Z}{A}\right)^{2}\right\} \\
& +V_{W}^{\prime}|N-Z| \exp \left\{-\left(\frac{A}{A_{0}}\right)^{2}\right\},
\end{aligned}
$$

and (ii) the rotational and vibrational spurious collective energy

$$
\begin{gathered}
E_{\text {coll }}=E_{\text {rot }}^{\text {crank }}\left\{b \tanh \left(c\left|\beta_{2}\right|\right)\right. \\
\left.+d\left|\beta_{2}\right| \exp \left\{-l\left(\left|\beta_{2}\right|-\beta_{2}^{0}\right)^{2}\right\}\right\}
\end{gathered}
$$

in which $E_{\text {rot }}^{\text {crank }}$ denotes the cranking-model value of the rotational correction [8] and $\beta_{2}$ the quadrupole deformation, while all other parameters are free fitting parameters. The correction term, Eq (4), differs from that used in our previous mass models. While the differences are small for large deformations, the collective energy given by Eq (4) now vanishes for spherical nuclei.

\section{Microscopically derived effective pairing force}

In all of our mass models HFB-1 to HFB-15, the density dependence of the effective pairing strength $v^{\pi q}\left[\rho_{n}, \rho_{p}\right]$ acting between nucleons of the same charge $q(q=n$ or $p$ for neutrons or protons, respectively) was parametrized by an expression of the usual following form

$$
v^{\pi q}\left[\rho_{n}, \rho_{p}\right]=V_{\pi q}\left\{1-\eta_{s}\left(\frac{\rho}{\rho_{0}}\right)^{\alpha}\right\},
$$

where $\rho=\rho_{n}+\rho_{p}$ is the total density, $\rho_{0}$ is the saturation density of symmetric nuclear matter, whereas $V_{\pi q}$ is a free parameter that were determined by the global mass fit. The surface parameters, $\eta_{s}$ and $\alpha$, were either set to zero assuming a purely volume pairing force or taken from the work of Ref. 9. Even when constraining the pairing strength $V_{\pi q}$ to a relatively low value in order to conform the nuclear pairing with odd-even mass differences, we found that our earlier mass models predict unrealistic pairing gaps in uniform nuclear-matter as compared to microscopic calculations while yielding comparably good mass fits (hence comparably good pairing gaps in finite nuclei).

In order to improve the treatment of pairing, we have recently developed a new series of HFB mass models by imposing the additional constraint of reproducing as a function of density the ${ }^{1} S_{0}$ pairing gaps of uniform asymmetric nuclear matter. Fitting all the model parameters while imposing this constraint would be an extremely onerous numerical task when using the ansatz (5). Instead of postulating a density dependence as in Eq. (5), we have thus determined the strength of the effective pairing force at each neutron and proton density by solving the HFB equations in uniform matter and requiring that the resulting gap reproduce exactly the microscopic pairing gap calculated with realistic forces at that neutron and proton density. In this way, the pairing strength for the nucleon species $q$ is given by

$$
\begin{gathered}
v^{\pi q}\left[\rho_{n}, \rho_{p}\right]=-8 \pi^{2}\left(\frac{\hbar^{2}}{2 M_{q}^{*}\left(\rho_{n}, \rho_{p}\right)}\right)^{3 / 2} \times \\
\times\left(\int_{0}^{\mu_{q}+\varepsilon_{\Lambda}} \mathrm{d} \xi \frac{\sqrt{\xi}}{\sqrt{\left(\xi-\mu_{q}\right)^{2}+\Delta_{q}\left(\rho_{n}, \rho_{p}\right)^{2}}}\right)^{-1}
\end{gathered}
$$

where $\Delta_{q}\left(\rho_{n}, \rho_{p}\right)$ is the corresponding pairing gap in uniform matter, $M_{q}^{*}\left(\rho_{n}, \rho_{p}\right)$ is the effective nucleon mass and $\varepsilon_{\Lambda}$ is the pairing cutoff. The chemical potential $\mu_{q}$ is approximated by

$$
\mu_{q}=\frac{\hbar^{2} k_{\mathrm{F} q}^{2}}{2 M_{q}^{*}}
$$

where $k_{\mathrm{F} q}=\left(3 \pi^{2} \rho_{q}\right)^{1 / 3}$ is the nucleon Fermi wave number.

Given the discrepancies between the various microscopic many-body calculations of the ${ }^{1} S_{0}$ pairing gaps in uniform nuclear matter, we have considered two different cases. In model HFB-16, we have taken the microscopic pairing gap calculated at the lowest BCS level using the Argonne v14 potential [10] and following Duguet [11], we have assumed that the pairing force for nucleons $q$ depends only on the density $\rho_{q}$. We have dropped this assumption in our latest model HFB-17 and we have considered the recent Brueckner calculations of Ref. 12 which include the effect on the interaction of medium polarization. These calculations were performed using the Argonne v18 potential both with and without self-energy corrections. Adopting a microscopic pairing gap calculated with self-energy corrections is quite challenging since ideally for consistency the Skyrme effective mass $M_{q}^{*}$ should be fitted to the corresponding microscopic effective mass. However this seems to be impossible within the framework of the conventional Skyrme forces used here. For this reason, we have used the microscopic pairing gap of Ref 12 including only medium-polarization effects and we have set $M_{q}^{*}=M$ in Eqs. (6) and (7).

Ref. 12 calculates pairing gaps only for symmetric nuclear matter, $\Delta_{S M}\left(\rho=\rho_{n}+\rho_{p}\right)$, and pure neutron matter, $\Delta_{N M}\left(\rho_{n}\right)$. Since we need the pairing gaps for arbitrary asymmetry we adopted the interpolation ansatz

$$
\Delta_{q}\left(\rho_{n}, \rho_{p}\right)=\Delta_{S M}(\rho)(1-|\eta|) \pm \Delta_{N M}\left(\rho_{q}\right) \eta \frac{\rho_{q}}{\rho}
$$

where $\eta=\left(\rho_{n}-\rho_{p}\right) / \rho$ and the upper (lower) sign is to be taken for $q=n(p)$; we have also assumed charge symmetry, i.e., $\Delta_{n}\left(\rho_{n}, \rho_{p}\right)=\Delta_{p}\left(\rho_{p}, \rho_{n}\right)$. This expression ensures that for symmetric nuclear matter, $\Delta_{q}(\rho / 2, \rho / 2)=$ $\Delta_{S M}(\rho)$ and for neutron matter $\Delta_{n}(\rho, 0)=\Delta_{N M}(\rho)$ and 
Table 1. Parameters of the analytical fit for the microscopic reference pairing gaps (the unit of length is fermi and the unit of energy is $\mathrm{MeV}$ ).

\begin{tabular}{|c|c|c|c|c|c|}
\hline & $\Delta_{0}$ & $k_{1}$ & $k_{2}$ & $k_{3}$ & $k_{\mathrm{m}}$ \\
\hline $\mathrm{SM}$ & 133.779 & 0.943146 & 1.52786 & 2.11577 & 1.51 \\
\hline $\mathrm{NM}$ & 14.9003 & 1.18847 & 1.51854 & 0.639489 & 1.52 \\
\hline
\end{tabular}

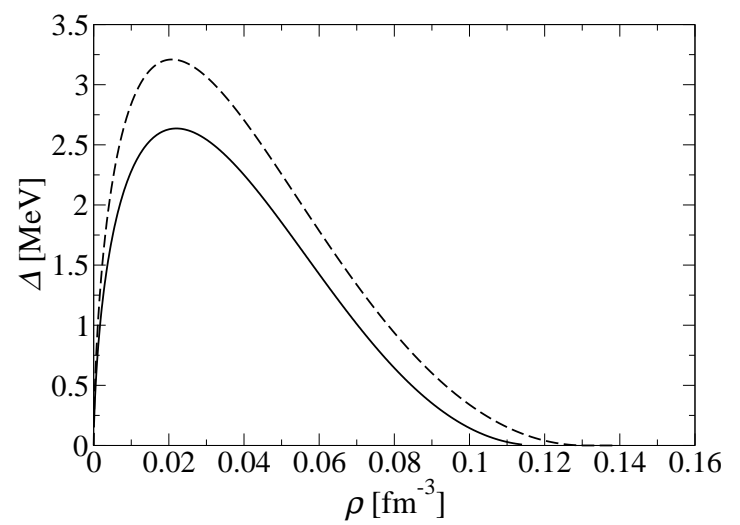

Fig. 1. ${ }^{1} S_{0}$ neutron pairing gap $\Delta$ in infinite neutron matter as a function of the density $\rho$. The dashed line represents the gap obtained in the BCS approximation from Ref. 10. and used in model HFB-16, while the solid line is the gap calculated in Ref. 12 including the effect on the interaction of medium polarization and used in model HFB-17.

$\Delta_{p}(\rho, 0)=0$. For convenience, we have used the essentially exact analytical representations

$$
\begin{gathered}
\Delta_{\mathrm{SM}}(\rho)=\theta\left(k_{\mathrm{m}}-k_{\mathrm{F}}\right) \Delta_{0} \frac{k_{\mathrm{F}}^{3}}{k_{\mathrm{F}}^{2}+k_{1}^{2}} \frac{\left(k_{\mathrm{F}}-k_{2}\right)^{2}}{\left(k_{\mathrm{F}}-k_{2}\right)^{2}+k_{3}^{2}}, \\
\Delta_{\mathrm{NM}}\left(\rho_{n}\right)=\theta\left(k_{\mathrm{m}}-k_{\mathrm{F} n}\right) \Delta_{0} \frac{k_{\mathrm{F} n}^{2}}{k_{\mathrm{F} n}^{2}+k_{1}^{2}} \frac{\left(k_{\mathrm{F} n}-k_{2}\right)^{2}}{\left(k_{\mathrm{F} n}-k_{2}\right)^{2}+k_{3}^{2}},
\end{gathered}
$$

where $k_{\mathrm{F}}=\left(3 \pi^{2} \rho / 2\right)^{1 / 3}, \theta$ is the Heaviside unit-step function, and the associated parameters are given in Table 1. The different pairing gaps are shown in Figs. 1 and 2 , For odd-nucleon nuclei we make use of the "equal-filling" approximation [13]. In order to take phenomenologically into account the contribution to pairing of the time-odd fields [14, we multiply the pairing strength by a parity factor $f_{q}^{ \pm}$( + for an even number of nucleons, - otherwise) that we allow to be different for neutrons and protons due to Coulomb forces and possible charge symmetry breaking effects. By definition we set $f_{n}^{+}=1$.

The corresponding effective pairing force, as given by Eq. (6), is shown in Figs. 3 and 4 for the HFB-17 mass model.

\section{Results of the mass fit}

As explained in the previous sections, the HFB-17 mass model [5] represents our latest attempt to provide a universal force capable of predicting globally static nuclear

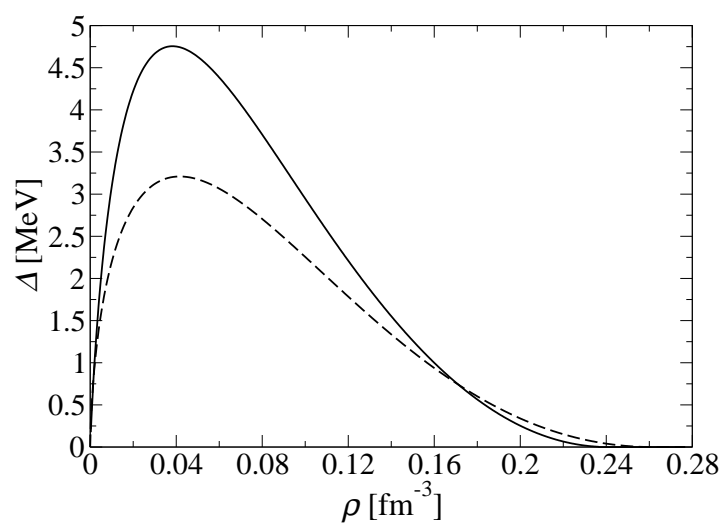

Fig. 2. ${ }^{1} S_{0}$ neutron pairing gap $\Delta$ in infinite symmetric nuclear matter as a function of the density $\rho$. The dashed line represents the gap obtained in the BCS approximation from Ref. [10] and used in model HFB-16, while the solid line is the gap calculated in Ref. 12 including the effect on the interaction of medium polarization and used in model HFB-17.

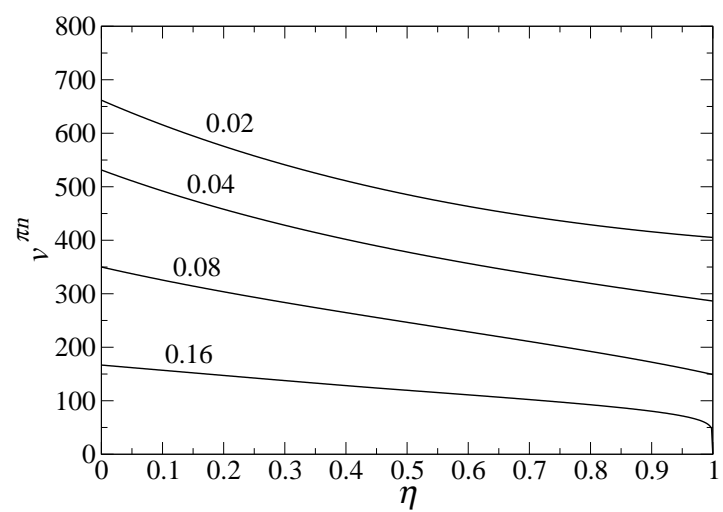

Fig. 3. Isospin dependence of the effective neutron pairing strength $v^{\pi n}$ of model HFB-17 for different densities $\rho$ (indicated above each curve in $\mathrm{fm}^{-3}$ ).

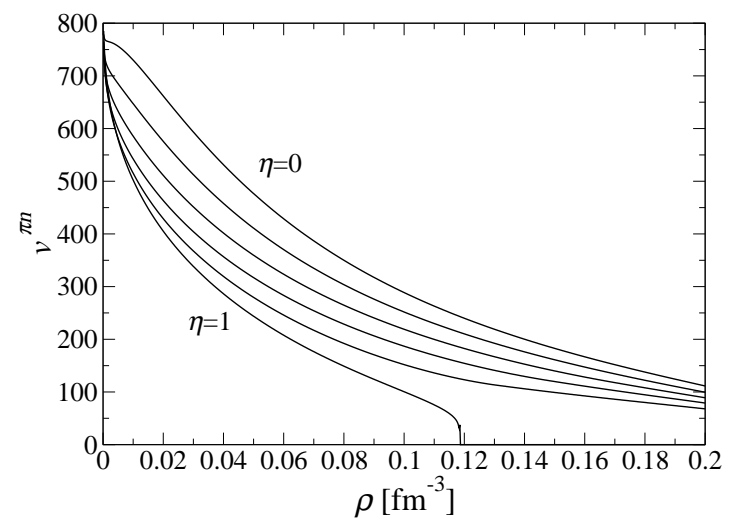

Fig. 4. Effective neutron pairing strength $v^{\pi n}$ of model HFB17 as a function of density $\rho$ for different isospin asymmetries $\eta$ by step of 0.2 . 


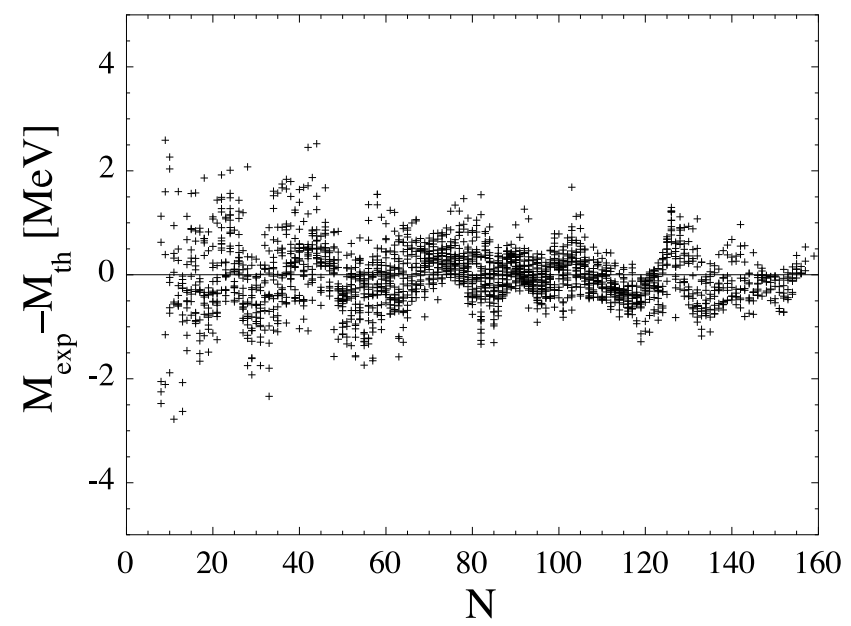

Fig. 5. Differences between experimental and calculated masses as a function of the neutron number $N$ for the HFB-17 mass model.

structure properties. The 10 parameters of the Skyrme force along with the 4 parameters of the pairing force (including the pairing cutoff) and the 9 parameters of the Wigner and collective corrections have been fitted on the 2149 measured masses of nuclei with $N$ and $Z \geq 8$ given in the 2003 AME [15. The deviations between the experimental data and the HFB-17 predictions are shown graphically in fig. 5. The rms and mean (data - theory) values of these deviations are $0.581 \mathrm{MeV}$ and $-0.019 \mathrm{MeV}$, respectively. HFB-17 is not only the most accurate mass model ever achieved within the mean-field framework, it also satisfies extra physical constraints that make it more suitable for astrophysics applications. In particular, the calculated quadrupole moments, charge radii, charge-density distributions and spins are also found to be in good agreement with experiment. As done in our previous mass fits, when determining the Skyrme force parameters, a special attention has been paid to properly describe the properties (not only the pairing) of infinite nuclear and neutron matter determined from realistic calculations $3,16,17$. The excellent fit of HFB-17 to the neutron matter curve of Ref. [3] was achieved simply by imposing a nuclear-matter symmetry coefficient of $J=30 \mathrm{MeV}$. The potential energy per particle determined with the BSk17 Skyrme force was also found to be in fair agreement with the recent calculations of Ref. [16] in each of the four two-body spin-isospin $(S, T)$ channels and for all densities. Finally, in contrast to most of the traditional Skyrme forces, our BSk17 (and previous forces) is consistent with the isovector splitting of the effective mass deduced from measurements of isovector giant resonances and confirmed in several many-body calculations with realistic forces [17. More details on these comparisons can be found in Ref. [5].

In Fig. 6 we compare the HFB-17 predictions with those of the finite range droplet model (FRDM) [18 for all $8 \leq Z \leq 110$ nuclei lying between the proton and neutron drip lines. Significant differences (up to $25 \mathrm{MeV}$ ) are found, especially for heavy neutron-rich nuclei. Such dif-

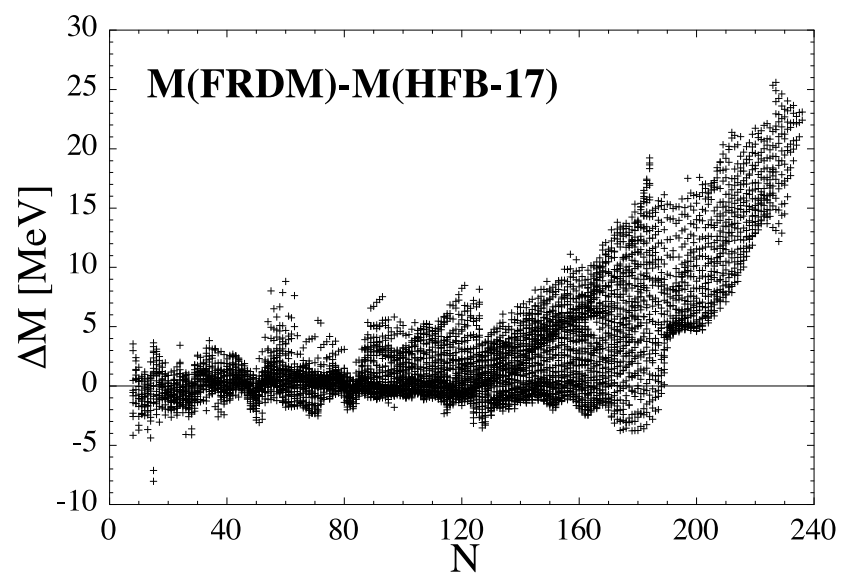

Fig. 6. Differences between the FRDM and HFB-17 mass predictions as a function of (left) $N$ for all $8 \leq Z \leq 110$ nuclei lying between the proton and neutron drip lines.

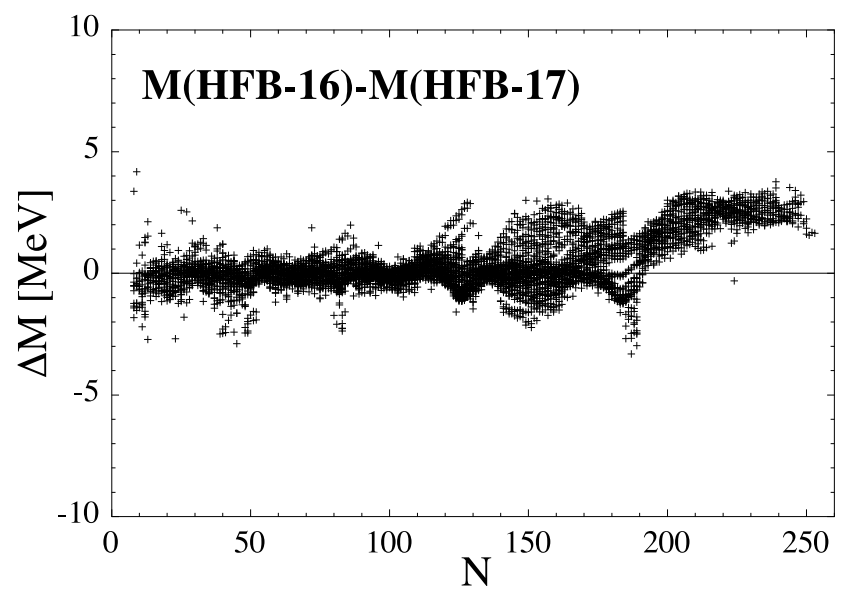

Fig. 7. Differences between the HFB-16 and HFB-17 mass predictions as a function $N$ for all $8 \leq Z \leq 110$ nuclei lying between the proton and neutron drip lines.

ferences are much larger than what is obtained between our various HFB mass models, as illustrated in Fig. 7

In Figs. 8 and 9, we show the neutron-shell gaps, defined by

$$
\Delta_{n}\left(N_{0}, Z\right)=S_{2 n}\left(N_{0}, Z\right)-S_{2 n}\left(N_{0}+2, Z\right),
$$

as a function of $Z$ for the magic numbers $N_{0}=50,82,126$ and 184 for the HFB-17 model ( $S_{2 n}$ is the two-neutron separation energy). Likewise in Fig. 10 we show the protonshell gaps as a function of $N$ for $Z_{0}=50$ and 82. Here we consider just the comparison with the data. Given the overall close agreement between experimental and theoretical shell gaps, the differences found for the $N_{0}=126$ neutron-shell gaps clearly emerge as unsatisfactory, especially in the vicinity of doubly-magic ${ }^{208} \mathrm{~Pb}$ nuclei. It is even more disturbing that the $Z_{0}=82$ proton-shell gaps agree rather well. The double magicity around ${ }^{208} \mathrm{~Pb}$ remains unresolved in all mean field calculations, regardless of the type of force or its parametrization. 


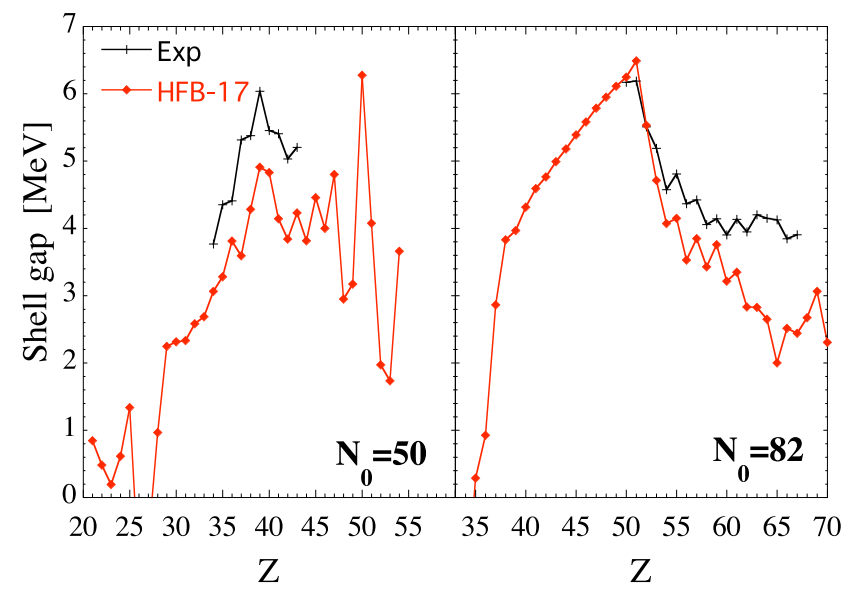

Fig. 8. $N_{0}=50$ and $N_{0}=82$ shell gaps as function of $Z$ for the mass models HFB-17.

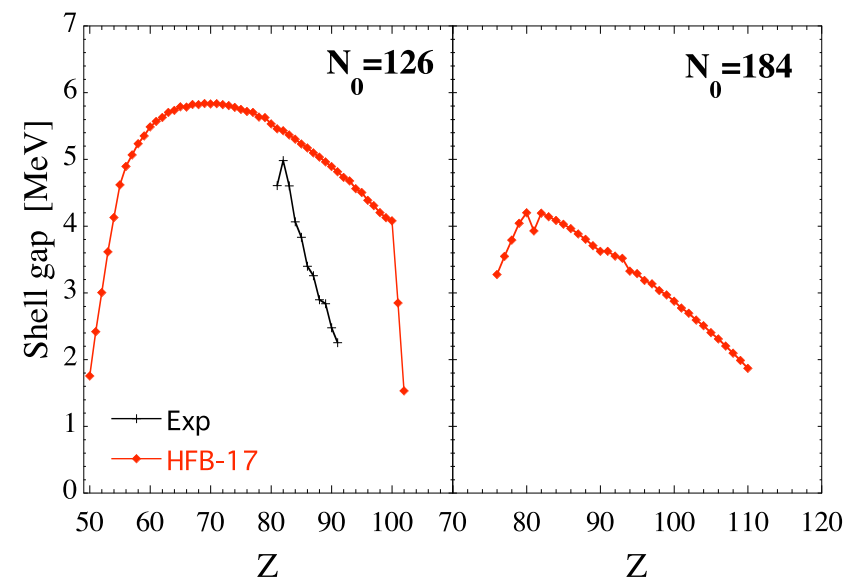

Fig. 9. $N_{0}=126$ and $N_{0}=184$ shell gaps as function of $Z$ for the mass models HFB-17.

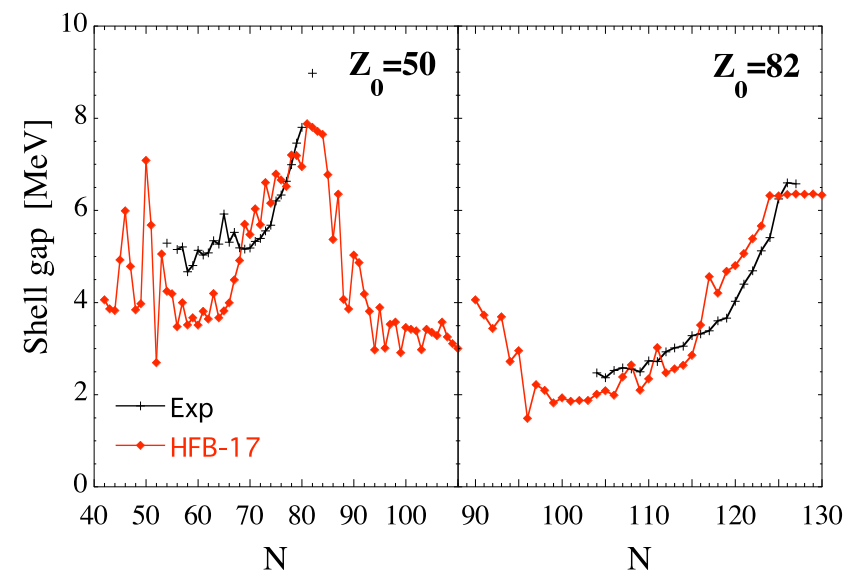

Fig. 10. $Z_{0}=50$ and $Z_{0}=82$ shell gaps as function of $N$ for the mass models HFB-17.

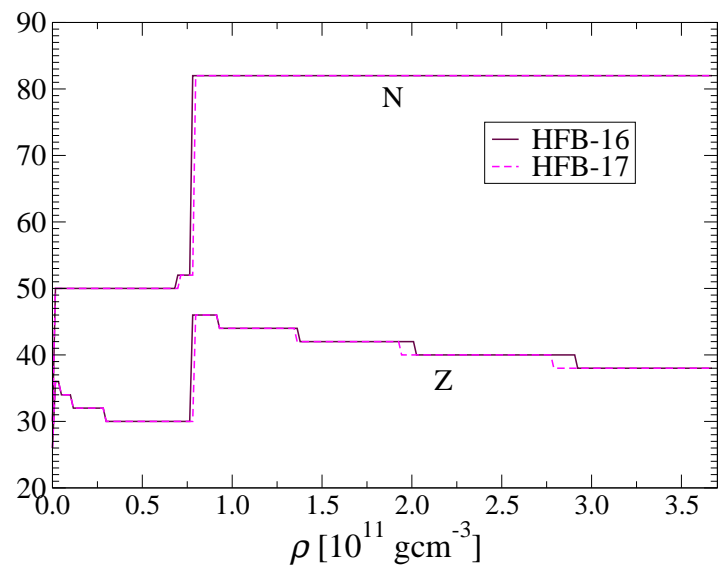

Fig. 11. Proton number $Z$ and neutron number $N$ of nuclei in the outer crust of neutron stars for the HFB-16 and HFB-17 mass models.

\section{Astrophysical applications}

Following the classical paper of Baym, Pethick and Sutherland [19, we have calculated the structure of the outer crust of neutron stars for the HFB-16 and HFB-17 mass models. The only microscopic inputs are the values of the atomic masses. We have used experimental data when available. The results are shown in Fig. 11. The composition of the nuclei in the outer crust is essentially the same for the two mass models HFB-16 and HFB-17. Both models predict that at densities above $\rho_{\mathrm{ND}} \simeq 3.7 \times 10^{11}$ $\mathrm{gcm}^{-3}$, neutrons start to drip out of nuclei. The corresponding layer of the outer crust is composed of nuclei with $Z=38$ and $N=82$.

The structure of the inner crust of neutron stars, at densities above $\rho_{\mathrm{ND}}$, is not simply determined by atomic masses due to the presence of the neutron gas. The free neutrons modify the properties of the "nuclei" by i) exerting a pressure on them and ii) by reducing their surface tension [1. In order to make reliable predictions of the composition of the neutron star crust beyond neutron drip, both the nucleons bound inside clusters and the free neutrons have to be described consistently. For this purpose, we have applied the Extended Thomas-Fermi method up to the 4th order (see Ref. 20] for details) with the Skyrme effective forces BSk16 and BSk17 underlying the HFB-16 and HFB-17 mass models respectively. As can be seen in Fig. 12, the two models yield very similar results. The properties of the neutron star crust (elastic constants, electric and thermal conductivities, etc.) are strongly dependent on the proton number $Z$ of the nuclear clusters. As shown in Fig. 13, the differences in $Z$ between the two crust models do not exceed 2 units.

\section{Conclusions}

Despite all the restrictions imposed on the model parameter space, we have obtained the most accurate mass table ever achieved within the mean field framework, the rms 


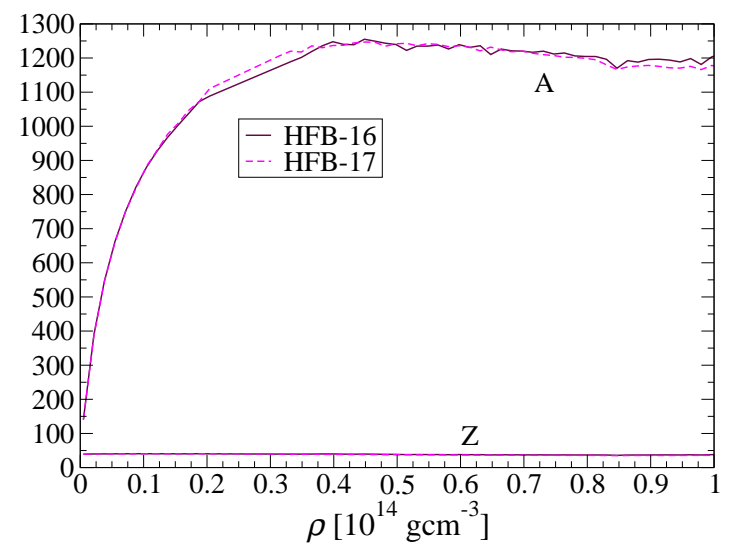

Fig. 12. Proton number $Z$ and mass number $A$ of "clusters" in the inner crust of neutron stars for the HFB-16 and HFB17 mass models. Note that $A$ includes not only the number of bound nucleons but also the mean number of free neutrons per lattice site.

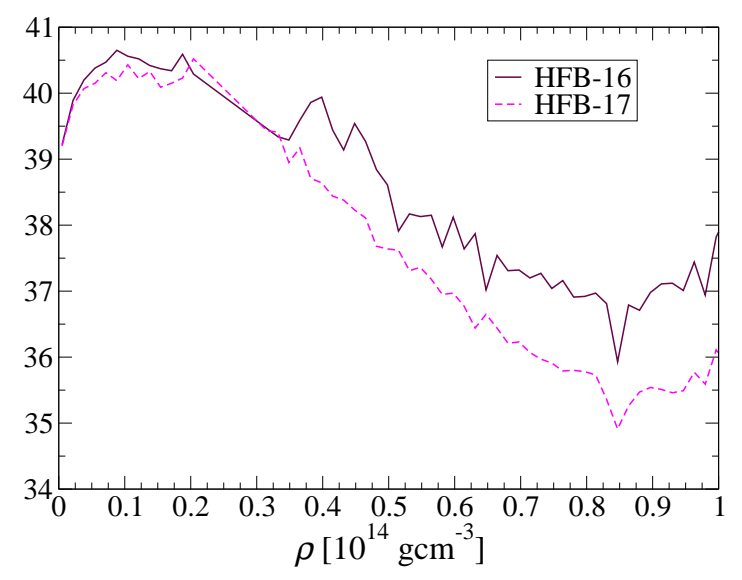

Fig. 13. Proton number $Z$ of nuclei in the inner crust of neutron stars for the HFB-16 and HFB-17 mass models.

deviation falling to $0.581 \mathrm{MeV}$. Given also the constraint imposed on the Skyrme force by microscopic calculations of nuclear and neutron matter, this new model is particularly well adapted to astrophysical applications involving a neutron-rich environment, such as the elucidation of the r-process of nucleosynthesis, and the description of supernova cores and neutron-star crusts. The HFB-17 mass table is made available to the scientific community at http://www-astro.ulb.ac.be

\section{References}

1. N. Chamel and P. Haensel, "Physics of Neutron Star Crusts", Living Rev. Relativity 11, (2008), 10. URL (cited on 15 December 2008): http://www.livingreviews.org/lrr-2008-10

2. S. Goriely, M. Samyn, J. M. Pearson, and M. Onsi, Nucl. Phys. A750 (2005) 425.

3. B. Friedman and V. R. Pandharipande, Nucl. Phys. A361, 502 (1981).

4. N. Chamel, S. Goriely, and J. M. Pearson, Nucl. Phys. A812, 72 (2008).
5. S. Goriely, N. Chamel and J. M. Pearson, accepted for publication in PRL (2008).

6. S.Goriely, F. Tondeur, J. M. Pearson, Atom. Data and Nucl. Data Tables 77, 311-381 (2001).

7. S. Goriely, M. Samyn, P.-H. Heenen, J. M. Pearson, F. Tondeur, Phys. Rev. C 66, 024326 (2002).

8. P. Tondeur, S. Goriely, J. M. Pearson, M. Onsi, Phys. Rev. 62, 024308 (2000)

9. E. Garrido, P. Sarriguren, E. Moya de Guerra and P. Schuck, Phys. Rev. C 60, 064312 (1999).

10. U. Lombardo and H. J. Schulze, "Superfluidity in neutron star matter" in LNP Vol. 578: Physics of Neutron Star Interiors, ed. D. Blaschke, N. K. Glendenning, and A. Sedrakian, Springer, 30 (2001).

11. T. Duguet, Phys. Rev. C 69 (2004) 054317.

12. L.G. Cao, U.Lombardo, and P.Schuck, Phys. Rev. C 74, 064301 (2006).

13. S. Perez-Martin and L. M. Robledo, Phys. Rev. C 78, 014304 (2008).

14. G. F. Bertsch, C. A. Bertulani, W. Nazarewicz, N. Schunk, and M. V. Stoitsov, nucl-th/0812.0747.

15. G. Audi, A.H. Wapstra, and C. Thibault, Nucl. Phys. A729, 337 (2003).

16. Z.H. Li, U. Lombardo, H.J. Schulze, and W. Zuo, Phys. Rev. C77, 034316 (2008).

17. W. Zuo, U. Lombardo, H.-J. Schulze, and Z. H. Li, Phys. Rev. C 74, 014317 (2006).

18. P. Möller and J. R. Nix, At. Data Nucl. Data Tables 39, 213, (1988).

19. G. Baym, C. Pethick and P. Sutherland, Astrophys. J. 170 299 (1971)

20. M. Onsi, A. K. Dutta, H. Chatri, S. Goriely, N. Chamel, J. M. Pearson, Phys. Rev. C 77 (2008), 065805. 